\title{
A Review of Risk Assessment Methods for Power System
}

\author{
Liang Zhao ${ }^{1 *}$, Tianyang Mao ${ }^{1}$, Wen $\mathrm{Xu}^{2}$, Jingzhao Luan ${ }^{2}$, Jiangning $\mathrm{Wu}^{2}$, Guangyuan $\mathrm{Qi}^{2}$. \\ ${ }^{1}$ Faculty of Electronic Information and Electrical Engineering, Dalian University of Technology, Dalian 116024, \\ China \\ ${ }^{2}$ State Grid Dalian Electric Power Supply Company, Dalian 116021, China
}

\begin{abstract}
In view of the current social background of power system becoming more and more complicated, and the current research situation of related fields, this paper discusses the common methods of power system risk assessment, including the method of hierarchy analysis, Markov chain model, Monte Carlo method, fuzzy comprehensive evaluation and related research progress. The advantages and disadvantages of the four methods are enumerated respectively. It is considered that the power system should be evaluated synthetically in the practical application and the effective risk assessment method should be adopted rationally.
\end{abstract}

Keywords.power system; risk assessment; analytic hierarchy process; Markov chain model; Monte Carlo method; fuzzy comprehensive evaluation

\section{INTRODUCTION}

With the extensive application and popularization of information technology, the information system has gradually penetrated into all walks of life, and has played a significant role in promoting social development. The safe operation of information system has become a necessary guarantee for the normal development of society. And as an important social infrastructure, the normal operation of the power system is the primary condition of sustainable development of social economy. On the other hand, in the power system to achieve great networking benefits at the same time, also had to bear a greater potential risk. Especially with the advancement of power market reform, the change of management institutions and the participation of new members in the market, the uncertain factors that are difficult to control and its influence on the power grid are more profound, making the planning, operation and maintenance of power system and asset management face a great challenge. Therefore, the importance of power system risk research is becoming more and more obvious.

An et al (2009) [1] analyzes the current situation of power system operation risk, power system equipment risk, power market risk and power system security risk, and points out that electric power workers in the system operation, equipment management, safety assessment and market analysis of these areas Research ideas change from deterministic research to uncertainty research. Zhang et al (2014) [2] analyzes the current situation of the research on the vulnerability of power system, and 
thinks that the current evaluation method can not meet the needs of modern power system, and it needs a lot of in-depth research. Liu et al (2016) [3] introduces the management system, database structure, index establishment principle and classification method of the reliability evaluation system of the large power system based on the operational data in North America, and explains the calculation and analysis method of the index.

Based on the present research situation of power system risk assessment at home and abroad, this paper introduces several risk assessment methods commonly used in power system, and analyzes their advantages and disadvantages.

\section{COMMON RISK ASSESSMENT METHOD FOR POWER SYSTEM}

\subsection{Analytic Hierarchy Process}

The analytic hierarchy process (AHP) is used to decompose the decision problem into different hierarchical structures according to the overall goal, the subordinate goals, the evaluation criteria and the specific alternatives. Then, the method of solving the matrix vector is obtained. The final weight of each element of each level is given to the priority of the previous level, and the final weighting method is the final weight of the candidate scheme to the total target. The final weight is the optimal scheme. The analytic hierarchy process is more suitable for the target system with hierarchical evaluation index, and the target value is difficult to quantitatively describe the decision problem. Its use is to construct the judgment matrix, find its maximum eigenvalue. And its corresponding eigenvector $\mathrm{W}$, after normalization, is a certain level of indicators for the previous level of a relative importance of the relative importance of indicators.

Based on the multi - factor analysis of large - scale blackout events, Wang et al (2011) [4] set up a set of safety and risk assessment system of power system with objectivity, practicability and applicability by using fault tree analysis and analytic hierarchy process. And the evaluation system is used to evaluate the power system of the provincial power system. The evaluation results validate the feasibility of the assessment methodology and the assessment indicators. Feng et al (2013) [5] applied 3 scale analytic hierarchy process assessment model for the establishment of the state of the transformer. Case analysis proved that assessment of power transformer condition based on Analytic Hierarchy Process method is reasonable and effective. Zhang et al (2013) [6] uses the 3-scale AHP method to derive the comprehensive risk of components through over-voltage risk, low-voltage risk, line overload risk, transformer overload risk and loss-of-load risk. And through the IEEE RTS-79 system to verify the risk based on the power system component risk assessment approach is feasible. Yang et al (2013) [7] use the analytic hierarchy process and the fuzzy comprehensive rating method to recalculate the expert evaluation weight for the current situation of the subjective factors of the expert in the risk assessment of the current power information system. The method is used to evaluate the components in the power information system. The results show that the method can reduce the subjective judgment factors. He (2016) [8] analyzes the current situation and existing problems of power information system construction, and uses the analytic hierarchy process to identify the main risks of power information system construction project. The research found that the risk of construction project of power enterprise information system mainly focused on the project stage and the realization stage, which provided the help for the future construction. In view of the shortcomings of the information security risk assessment mechanism in traditional power systems, such as poor accuracy, lack of perfection and low efficiency, Liang et al (2017) [9] introduce the analytic hierarchy process into the risk assessment mechanism according to the specific application of the power system. They use the fuzzy mathematics knowledge in the process of risk calculation to design a new model of information security risk assessment, namely AF-RA model, and elaborate and analyze the model.

\subsection{Markov Chain Model}

Markov chain is a stochastic process of discrete events with Markovian properties in mathematics. In the 
process, in the case of a given current knowledge or information, the past is irrelevant for the prediction of the future. The Markov chain describes a state sequence whose state values depend on the previous finite state. The Markov chain is a sequence of random variables with Markovian properties. The range of these variables, that is, their all possible sets of values, is called "state space" and the value of $\mathrm{Xn}$ is in the state of time $\mathrm{n}$. If $\mathrm{Xn}+1$ is $\mathrm{Xn}$ function of the conditional probability distribution for the past state, then:

$$
P\left(X_{n+1}=x \mid X_{1}=x_{1}, X_{2}=x_{2}, \ldots, X_{n}=x_{n}\right)=P\left(X_{n+1}=x \mid X_{n}=x_{n}\right) \cdot(1)
$$

Where $\mathrm{x}$ is a state in the process. The above identity can be regarded as the Markovian nature.

Guo et al (2012) [10] define an improved Markov chain, the peak-type Markov chain, for the defects of traditional Markov chains in some special cases of power systems, and prospects its application in the power system. Magnus et al (2012) [11] proposed a new method for generating samples of the power at all nodes given a set of transfers through specified corridors of the power system. In the reliability analysis of power system, the Monte Carlo simulation method requires a large number of computational resources and the slow convergence speed. From the operational point of view, based on the Markov chain, Wang et al (2013) [12] proposed a model for rapid analysis of power system reliability. The validity of the study is proved by the analysis of the examples. Tang et al (2013) [13] proposed a refinement pattern recognition method for transient stability assessment of power systems based on a two-stage parallel Hidden Markov model. The simulation and analysis of the actual regional grid environment verify the effectiveness and accuracy of the method. In the case of recognition accuracy, this method is less than the training samples required for commonly used artificial intelligence methods (such as ANN, SVM, etc.) and converges faster. In the reliability analysis of power system, the shortcomings of long time residence time in the same state are often simulated by using the discrete Markov chain model, based on the continuous time Markov chain, Jin et al (2015) [14] propose a model and give an example of the proposed research method. Based on the hidden Markov model, Ma et al (2016) [15] improve the existing static risk assessment algorithm based on the influence of entity behavior on system risk.
This paper analyzes the system assets, threats and vulnerabilities, and presents an improved risk calculation method. Theoretical analysis and experimental results show that the improved method improves the reliability and timeliness of the evaluation results. Mindaugas et al (2016) [16] proposed stochastic automata networks to allow reducing the size of state space of Markov chain model and simplifying system specification. Modelling results showed that the implementation of Markov chain model by using SAN method is a relatively easy task.

\subsection{Monte Carlo Method}

Monte Carlo method, also known as statistical simulation method, random sampling technology, is a stochastic simulation method, based on probability and statistical theory method is a calculation method, is the use of random numbers to solve a lot of computing problems. The problem is solved by a certain probability model, and the statistical simulation or sampling is realized by an electronic computer to obtain the approximate solution of the problem.

Bie et al (1997) [17] introduce the basic principles of Monte Carlo method and its application in power system reliability assessment. Through the comparison and analysis of Monte Carlo method and analytic method, the characteristics of Monte Carlo method and its application range are expounded. Then, the application status of Monte Carlo method in power system reliability evaluation is introduced from the aspects of improving the convergence speed of Monte Carlo method, the combination of Monte Carlo method and analytic method, pointing out the future research direction. In order to comprehensively evaluate the economics and reliability of wind power generation, Wu et al (2004) [18] proposed a reliability model of wind turbine based on sequential Monte Carlo simulation, and gave a detailed algorithm, taking the random characteristics of wind energy and the forced outage of wind turbines into account. The model is used to simulate the wind turbine and the time series load model is used to calculate the system reliability index and economic index under different design schemes. Simona et al (2007) [19] presented the development of a reliability simulation program for electrical power stations based on Monte Carlo method. 
The program can help in equipment and system condition evaluation and monitoring. Based on the power system operational risk assessment system, based on the analysis and evaluation of the critical system of power system operation risk index set, Xia et al (2014) [20] use non-sequential Monte Carlo simulation technology quantitative analysis and comprehensive consideration of equipment failure probability and consequences of the risk index. They through the various control measures after the implementation of the changes in the risk indicators to assist the system operator decision-making, which has important engineering application value. Bao et al (2014) [21] analyzed the differences in the application of the two conventional sequential Monte Carlo simulations in the power system reliability assessment of the state duration sampling (SDS) and the time interval sampling (TIS). The validity of the proposed method is verified by the simulation of IEEE-RTS79 test system, which indicates that the modified TIS method has more obvious advantage when the system failure rate increases. Chen (2016) [22] first outlined the concept and basic theory of power system risk assessment, and then details the most commonly used Monte Carlo method in risk assessment, which is mainly divided into sequential Monte Carlo method and non-sequential Monte Carlo method. This paper uses the data of Hainan power grid to carry out the risk assessment analysis. The risk assessment results verify the validity and practicability of the power system risk analysis in the power system.

\subsection{Fuzzy Comprehensive Evaluation}

Fuzzy comprehensive evaluation method is a comprehensive evaluation method based on fuzzy mathematics. The comprehensive evaluation method is based on the membership degree theory of fuzzy mathematics to transform the qualitative evaluation into quantitative evaluation, that is, using fuzzy mathematics to make a general evaluation of things or objects subject to various factors. It can better solve the problem which is fuzzy, difficult to quantify, suitable for a variety of non-deterministic problems.

Daniela et al (2001) [23] presented practical applications of fuzzy sets methods to power systems control, design, operation and management, and demonstrated that fuzzy methods can solve many difficult problems. Liang et al (2009) [24] put forward a comprehensive evaluation model and method of information system security risk based on fuzzy comprehensive evaluation theory, and realized the goal of quantifying the security risk of information system. Based on the set of safety risk factors, the set of weights and the weight coefficient of the information system, the fuzzy risk comprehensive evaluation matrix is established and applied to the security risk assessment of the Web components of the power information system. $\mathrm{Wu}$ et al (2011) [25] proposed the concept of "relevant fuzzy number" and "independent fuzzy number" for the deficiency of fuzzy operation defined by expansion theorem, and an improved method suitable for fuzzy operation is proposed in combination with the principle of expansion, and the rationality of the method is proved. The fuzzy uncertainty of equipment outage and load level under different operating conditions of power system is analyzed, and the fuzzy model of equipment outage and multi - level load level is established. The risk assessment procedure and risk index of power system based on improved fuzzy operation are given. Finally, taking the IEEE - RTS 24 - node test system as an example, the improved fuzzy operation method is used to calculate the specific system risk index, and the results are compared and analyzed. The correctness and rationality of the method are verified. Based on the research of the theory of information fusion system and the security characteristics of power system, Song (2014) [26] designs the quantitative risk assessment method of power information physical fusion system by introducing the comprehensive fuzzy risk assessment method. Using the fuzzy set theory, the triangular fuzzy number is used to carry out the risk data transformation, and the parameters are integrated to calculate the overall risk level of the system. Tan (2014) [27] studied the fault probability grading algorithm based on fuzzy mean clustering method, and proposed the grading algorithm of fault consequence severity based on fuzzy reasoning principle, and established the safety warning level by fuzzy operation. The wind speed prediction model based on time series state transition method and the output model and outage model of wind turbine with 
consideration of wake effect are established. Based on fuzzy synthetic evaluation method, Wu et al (2017) [28] assessed the risks in public-private partnership straw-based power generation projects in China. This study expands the knowledge and contributes to the literature.

\section{ANALYSIS ON ADVANTAGES AND DISADVANTAGES OF POWER SYSTEM EVALUATION METHOD}

The analytic hierarchy process has the advantages of systematic analysis method, simple and practical decision-making method and less quantitative data information. But also can not provide new solutions for decision-making, less quantitative data, more qualitative components, not convincing, too many indicators when the data statistics, and the weight is difficult to determine the eigenvalues and eigenvectors of the exact method of more complex shortcomings.

The Markov chain model has a good effect on the state prediction of the process, and can be used to predict the production of dangerous state, but it is not suitable for long-term prediction.

Monte Carlo method can simulate the characteristics of things with random properties and physical experimental process, subject to the constraints of geometric conditions is small, the convergence rate has nothing to do with the dimension of the problem, in dealing with multi-dimensional problems have more obvious advantages, With the ability to simultaneously calculate multiple solutions with multiple unknowns, the error is easy to determine, the program structure is simple, easy to use computer to achieve the advantages. At the same time, it has the shortcomings of slow convergence and error.

Fuzzy evaluation through accurate digital means to deal with fuzzy evaluation of the object, can be hidden information on the information presented by the more scientific, reasonable, close to the actual quantitative evaluation. And the evaluation result is a vector, rather than a point value, contains a wealth of information, both can accurately describe the object to be evaluated, but also can be further processed to get reference information. But the calculation is complex, and the subjective weight of the index weight vector is strong.

\section{CONCLUSION}

The above article introduces five common methods of risk assessment of power systems and compares their advantages and disadvantages. It can be seen that each method has to be in its appropriate environment to play the greatest effect. In the actual production, according to the actual situation, a reasonable choice of assessment methods, if necessary, can be used in conjunction with multiple methods to achieve rapid and accurate risk assessment.

\section{Acknowledgment}

The present research is supported by the national science and technology Power Grid Corp (2017YF-36).

\section{Reference}

1. T.-Y. An, Z.-Y. Wang, X.-Z. Jin, J.-N. Zhang and H. Hu, Power System and Clean Energy 9, pp.4-10, September(2009)

2. X.-D. Zhang, R.-K. Sun, P. He and S. Zhang, The Proceedings of the 8th Annual Conference of Science and Technology of Jilin Province, China. (2014)

3. W.-X. Liu, X.-D. Shan, W.-M. Jiang, Z.-Q. Wang and B.-L. Xia, Power System Technology 40, pp. 3811-3817, December(2016).

4. B. Wang, D.-H. You, X.-G. Yin and Q.-Q. Chen, Power System Technology 35, pp.40-45, January (2011)

5. Y. Feng, Z.-H. Zhang and L.-Z. He, Advanced Materials Research 805-806, pp. 793-800(2013)

6. Y.-M. Zhang, Z.-H. Zhang, F. Yao, L.-Z. He and W. Xu, Power System Protection and Control 41, pp.73-78, December(2013)

7. H. Yang, Y. Wang, X.-G. Pei and S. Chen, Journal of Shanghai University of Electric Power 29, pp.518-522, December(2013)

8. Y.-M. He, North China Electric Power University, (2016)

9. Z.-Q. Liang and D.-S. Lin, Netinfo Security 4, 
pp.86-90(2017)

10. H.-K. Guo and S.-K. Heng, Journal of Electric Power 27, pp.96-98, April(2012)

11. M. Perninge and L. Soder, European Transactions on Electrical Power 22, pp.140-151, March(2012)

12. Y. Wang, X.-S. Han, Y. Ding and J. Sheng, Power System Technology 37, pp.405-410, February( 2013)

13. F. Tang, B. Wang, X.-M. Zha, Z.-H. Ma and Y.-N. Shao, Proceedings of the CSEE 33, pp.90-97, April( 2013)

14. S.-H. Jin, J.-G. Lu, Y.-P. Wan, S.-G. Sun and J.-P. Liu, Fifth International Conference on Reliability and Electrical Contact for Electrical Products, China(2014)

15. Z.-C. Ma, P. Yang, X.-F. Zhang and L.-T. Ding, Modern Electronic Technique 39, pp.165-170, September(2016)

16. M. Snipas, V. Radziukynas and E. Valakevicius, Reliability Engineering and System Safety 157, pp.13-22, January(2017)

17. C.-H. Bie and X.-F. Wang, Automation of Electric Power Systems 22, pp.68-75, June(1997)

18. Y.-C. $\mathrm{Wu}$ and M. Ding, Electric Power Automation Equipment 24, pp.70-73, December( 2004)

19. S. Dzitac, T. Vesselenyi, I.Dzitac and E. Valeanu,
The 4th International Federation of Automatic Control Conference on Management and Control of Production and Logistics, September(2007)

20. T. Xia, T.-G. Zhou, X. Zhang, Y. Wang and G.-J. Liu, Journal of State Grid Technology College 17, pp.1-21(2014)

21. Y.-K. Bao, Y. Wang, J.-X. Tang, C.-X. Guo, X. Wang and H. Shi, Power System Technology 38, pp.1189-1195, May(2014)

22. Z. Chen, Technology Innovation and Application. pp.9-10(2016)

23. D. Andone, R. Dobrescu and S. S. Iliescu, IFAC Proceedings Volumes 34, pp.151-156, July(2001)

24. D.-X. Liang and X. Chen, Power System Protection and Control 37, pp.61-64, $\operatorname{March}(2009)$

25. H.-B. $\mathrm{Wu}$ and J. Hu, Journal of North China Electric Power University 38, pp.34-39, November(2011)

26. C.-Y. Song, Electronic Technology \& Software Engineering. pp.261(2016)

27. S.-M. Tan, North China Electric Power University, (2014)

28. Y.-N. Wu, L.-W.-Y. Li, R.-H. Xu, K.-F. Chen and Y. Hu, Journal of Cleaner Production 161, pp.977-990, September(2017) 